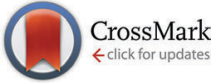

Cite this: Phys. Chem. Chem. Phys., 2015, 17, 17380

Received 23rd April 2015, Accepted 29th May 2015

DOI: $10.1039 / c 5 c p 02379 c$

www.rsc.org/pccp

\title{
Complexes of peracetylated cyclodextrin in a non-aqueous aprotic medium: the role of residual water $\dagger$
}

\author{
Laszlo Jicsinszky, ${ }^{* a}$ Katia Martina, ${ }^{a}$ Marina Caporaso, ${ }^{a}$ Pedro Cintas, ${ }^{b}$ \\ Andrea Zanichellic and Giancarlo Cravotto*a
}

\begin{abstract}
This paper describes the interaction between aromatic esters and peracetylated cyclodextrins (CDs) studied by NMR spectroscopy in deuterochloroform $\left(\mathrm{CDCl}_{3}\right)$. The observed chemical shift changes highlight the existence of interactions between an aromatic alkyl ester, water and peracetylated CDs. In some cases, substituent chemical shift determination was influenced by the low water content of $\mathrm{CDCl}_{3}$ and/or the host molecule. Higher CD concentrations resulted in water signal drifts in all studied cases. It was not possible to obtain a completely dry sample of peracetyl $\gamma C D$ : $\sim 1$ mol of water remained and the water signal showed reversed movement, with respect to the other two CD analogues, upon increasing host concentration. The estimated 1:1 stability constants for the water:peracetyl CD complexes are in the $50-150 \mathrm{M}^{-1}$ range in $\mathrm{CDCl}_{3}$, but show a relatively large calculation error. The calculated 1:1 stability constants for the peracetyl $C D$ : ester complexes are also in this range, but $1: 2$ and 2:1 complex compositions are also possible. Overall, our results highlight dynamic aspects of water nanoconfined in a highly hydrophobic environment, thus mimicking biological recognition where a few water molecules often play a pivotal role.
\end{abstract}

\section{Introduction}

Cyclic maltooligosaccharides, commonly known as cyclodextrins (CDs), are macrocycles of $\alpha(1 \rightarrow 4)$ linked anhydroglucopyranoside units in a ${ }^{4} \mathrm{C}_{1}$ conformation. In practice, the 6-, 7- and 8-membered versions are known as $\alpha$-, $\beta$-, and $\gamma$-cyclodextrins $(\alpha \mathrm{CD}, \beta \mathrm{CD}$, and $\gamma \mathrm{CD})$, respectively, and have all been well studied. Their low commercial price, particularly when bought in bulk, means that they are used worldwide in a variety of fields. The circular hydrogen bonds between the secondary hydroxyl groups on adjacent rings leads to a rigid truncated cone shape, where the hydroxyls form a highly hydrophilic rim and the glycosidic oxygens, together with a number of methine groups, create a considerably less hydrophilic environment inside the cavity. However, long-term inclusion normally occurs and rigid

\footnotetext{
${ }^{a}$ Dipartimento di Scienza e Tecnologia del Farmaco, University of Turin, Via P. Giuria, 9, 10125, Turin, Italy. E-mail: ljicsinszky@gmail.com, giancarlo.cravotto@unito.it

${ }^{b}$ Departamento de Química Orgánica e Inorgánica, Facultad de Ciencias-UEX, Avda. de Elvas s/n, E-06006 Badajoz, Spain

${ }^{c}$ Resilia SRL, Via Milano 201 - 21017, Samarate, VA, Italy

$\dagger$ Electronic supplementary information (ESI) available: ${ }^{1} \mathrm{H}$ NMR spectra of pure compounds and complexes at low, middle, and high peracetyl CD molar ratios, tables of proton chemical shifts, and figures of calculated concentrations and chemical shifts. See DOI: 10.1039/c5cp02379c
}

symmetric structures are frozen in the crystalline state only, in solutions a dynamic equilibrium exists between association and dissociation. Native CDs, except $\beta C D$, are easily soluble in water, while apolar guests easily displace the water molecules in the cavity via complexation resulting in a thermodynamically more favorable state. Inclusion complexes and the sandwichlike molecular associations are also often stabilized by hydrogen bonds.

Once all hydroxyls are substituted, particularly as alkyl ethers or esters, the CD lipophilicity profile is completely reversed and the cavity becomes more hydrophilic than the outer surfaces, while substitution also enlarges CD cavity dimensions. However, substituted oxygens, particularly glycosidic oxygens, are still able to form hydrogen bonds with appropriate hydrogen bond donors and lipophilic interactions become dominant, particularly at the edges of the enhanced cavities. It is also generally accepted that some smaller substituents, such as methyl or acetyl group, can considerably deform the structures in the absence of a guest, as has been described for peracetylated $\beta^{-1}$ and $-\gamma$-cyclodextrins ${ }^{2}$ (PAc $\beta C D$ and PAc $\gamma \mathrm{CD}$, respectively). The crystal structures of peracetyl- $\alpha$-cyclodextrin $(\mathrm{PAc} \alpha \mathrm{CD}),{ }^{3}$ and probably $-\beta \mathrm{CD},{ }^{4}$ are somehow symmetrical and stabilized by crystal water, although it is also true that these substituted CDs rarely reproduce the usual truncated cone structure of unsubstituted CDs. The more flexible structures obtained by breaking hydrogen bonds or 
even completely losing the hydrogen-bond belt mean that the glucopyranose rings are able to rotate around the glycosidic linkages. In fact, some isolated units have been found to distort from the usual ${ }^{4} \mathrm{C}_{1}$ chair conformation to skew or almost completely inverted ${ }^{1} \mathrm{C}_{4}$ conformers. ${ }^{1}$ The larger macrocycle in $\gamma \mathrm{CD}$ derivatives allows for greater flexibility and results in the most distorted crystal structures, as reported by Caira et al. ${ }^{2}$ Their solid-state structures have been described as elliptically distorted macrocycles where glucose units undergo significant rotation around the glycosidic connections and deform to create twisted conformers. ${ }^{4}$ Derivatization may not only create an enlarged cavity but can also lead to the self-inclusion of functional groups.

NMR techniques are currently the leading non-destructive methods for studying complexation processes in solution. Although various approaches have been formulated, two principal objectives are at the heart of this NMR titration based method. These two methodologies require two conflicting approximations. Determination of complex stoichiometry needs the sum of molar concentrations, a boundary condition for the most commonly used Job's plot method, to be constant. Calculation of complex stability constants, however, usually requires guest concentration being held constant in order to eliminate concentration dependent chemical shift drifts. ${ }^{5,6}$ In both cases, the equation for measured chemical shift $\left(\delta_{\text {obs }}\right)$, as a weighted average of the chemical shifts of the uncomplexed $\left(\delta_{\text {free }}\right)$ and complexed $\left(\delta_{\text {bound }}\right)$ species, is combined with the equation for the association constant according to complex stoichiometry. A comprehensive review by Uccello-Barretta et al. traces the development and historical milestones in NMR technology's study of cyclodextrin complexes. ${ }^{7}$

Several exhaustive reviews have been dedicated to the use of NMR methods on cyclodextrins and their complexes, ${ }^{5,8-10}$ while Chankvetadze has published a fundamental review ${ }^{11}$ on enantiodiscrimination studies. He also pointed out that the multiple forces involved in analyte-CD interactions mean that combined molecular modeling-spectroscopy approaches are required if we are to shed more light on the molecular basis of chiral recognition processes.

The presence of self-association phenomena can also be evaluated by analyzing the NMR spectra of the pure component in progressively diluted solutions; the self-association constant can be determined when chemical shifts depend on concentration. Preliminary information on intermolecular interactions can be obtained directly from an analysis of the complexation shifts $\left(\Delta \delta=\delta_{\text {obs }}-\delta_{\text {free}}\right)$, measured for all the species nuclei, in ${ }^{1} \mathrm{H}$ NMR spectra. However, the most important conformational analysis method when defining binding geometry is the detection of dipolar interactions (NOEs). ${ }^{5}$ This method furnishes information on the spatial proximity between nuclei and, therefore, highlights proximity constraints between nuclei that are either located inside the same molecule (intramolecular dipolar interactions) or belong to a different one (intermolecular dipolar interactions). This technique is also an important tool in chiral discrimination research, as intermolecular dipolar interactions make it possible to define the relative stereochemistry of the chiral auxiliary and one, or other, of the enantiomers in diastereoisomeric complexes. Both native and modified CDs are effectively used as chiral selectors in the majority of enantioseparation techniques. ${ }^{7,11}$

Although proton NMR measurements are the most common ways to investigate molecular associations, carbon NMR can also be used, but it is less feasible due to the less natural abundancy of NMR active ${ }^{13} \mathrm{C}$ and longer relaxation time of carbon. Other geometrical parameters, such as dihedral angles, can be determined using empirical relationships to analyze vicinal coupling constant dependence on dihedral angles. ${ }^{12,13}$ State-of-the-art high resolution NMR equipments also enable the scientists to study the effects of complexation on vicinal coupling constants. However, this effect is usually considerably less intense and more compound dependent than simple chemical shift analyses.

The enantioselective inclusion phenomena of lipophilic CD derivatives were reported in the pioneering work of König et al. ${ }^{14}$ who described the interaction between $(R, S)$-methyl-2chloropropionate and heptakis(3-O-acetyl-2,6-di- $O$-pentyl)- $\beta C D$ (Lipodex D). NMR spectra recorded in cyclohexane- $\mathrm{d}_{12}{ }^{15-17}$ indicated that the guest enantiomeric methine proton displayed very high differentiation. Complexation shifts were in accordance with GC retention data and a molecular dynamics simulation based chiral recognition rational was devised. Numerous publications that used regiospecific acetylated CDs, mainly per(6-TBDMS-2,3-di-O-acetyl) CDs, then appeared. ${ }^{15-17}$

While $\mathrm{H}-3$ and $\mathrm{H}-5$ protons are the most heavily involved in the inclusion processes in unsubstituted CDs, hydroxyl group substitution increases the number of moieties to be investigated. In addition, the resulting spectra sometimes show very complex chemical shift patterns which are due to the normally distorted structures in the un-complexed states discussed above. However, the spectra of unsubstituted and persubstituted CDs are much simpler than those of the partially derivatized compounds.

Persubstituted versions are the simplest of the acetylated CD derivatives to prepare. They can be easily produced even on a ten-hundred-kg scale and are available as fine chemicals as well. These CDs are highly lipophilic compounds and are soluble in various organic solvents such as methylene chloride, chloroform, toluene, ethyl acetate and acetone, for example. In toluene, they form inclusion complexes, which precipitate after dissolution of the CD. They are chemically inert and the acetyl groups cause the cavity of the cyclic structure to widen. The steric stress inherent in these macrocycles makes it possible for anhydrous acids or Lewis acids to cleave the glycosidic bonds, while acetyl groups can usually be cleaved under basic conditions, like in the majority of organic esters.

Although peracetylated CDs were among the first CDs to be prepared, ${ }^{18}$ their complexation properties have only been sporadically studied. However, the discovery that even polar compounds, such as organic alkali metal salts, can be complexed by PAc $\beta C D$, despite the cavity's reversed polarity, is not novel. ${ }^{19}$ This same phenomenon was also observed in peracetylated 3,6-anhydro-CDs. However it remained but a curiosity despite the possible advantages it may offer to the routinely 
used analytical method, TLC. ${ }^{20,21}$ Despite some attempts made to use these complexation properties in capillary GC, the unfavorably high melting point of peracetyl CDs $\left(\sim 235{ }^{\circ} \mathrm{C}\right.$, $\sim 198{ }^{\circ} \mathrm{C}$ and $\sim 248{ }^{\circ} \mathrm{C}$ for peracetyl- $\alpha-,-\beta$ - and $-\gamma \mathrm{CD}$, respectively) has been an obstacle to the wider penetration of these derivatives into the analytical repertoire. ${ }^{22}$

Enhanced lipophilicity and widened cavities would appear to make peracetylated CD use in the controlled release of hydrophilic drug obvious. Their potential has been known for 25 years, ${ }^{23}$ however after the initial advantageous attempts, only a few publications can be found. Some enantioseparations, both on analytical and preparative scales, ${ }^{24-28}$ have also been published and a couple of solid complexes have been prepared using various techniques. ${ }^{29-31}$

The investigation of soluble complexes in solution, particularly in apolar solvents, is rare and only a few examples are available in technical papers. ${ }^{4,19,24,32}$ Guest molecules in those cases also carry some polar components, apart from a species studied in a recent analytical impacted publication by Kopytin et al. ${ }^{22}$ The effect of $\alpha \mathrm{CD}$ peracylation on its molecular structure has been investigated using single crystal X-ray analysis, ${ }^{3}$ and only revealed the presence of at least two associated water molecules in the solid state, despite the crystalline complex being prepared from aqueous 2-propanol. Crystals of a heptakis(2,3,6tri-O-acetyl)- $\beta$ CD methanol complex, as well as other peracylated CDs, were found to contain elliptically distorted molecules with non-planar, boat-shaped structures. ${ }^{4}$ Steric interactions between adjacent acyl chains in these molecules result in the severe tilting of the glucose units relative to the glycosidic $\mathrm{O}(4)$ planes. Additionally, significant acyl residue self-inclusion was found in those peracylated molecules. This self-inclusion means that the guest inclusion, occurring in native host molecules, is unfavorable both in the solid state and in solution. The structure of fully acetylated $\gamma \mathrm{CD}$ also showed extensive macrocyclic distortion, in this case accompanied by the self-inclusion of three acetyl residues, while the complexation of alcohols occurred together with water molecules. ${ }^{2}$ A unique feature of the structure is the partitioning, by these residues, of the cavity region into two distinct sub-cavities that accommodate water and ethanol molecules. This unusual type of solvent dual inclusion was discovered thanks to powder X-ray diffraction experiments on PAc $\gamma \mathrm{CD}$ and a series of solvents, such as acetone, $n$-propanol and ethanol. ${ }^{2}$

The complete assignment of peracetylated $\beta C D$ signals has been carried out by Uccello-Barretta et al. who have also determined proximity constraints between PAc $\beta C D$ protons using 2D ROESY analysis in $\mathrm{CDCl}_{3}$ and $\mathrm{CD}_{3} \mathrm{OD}{ }^{1}$ It was also found that $\mathrm{H}-3, \mathrm{H}-2$ and $\mathrm{H}-6 \mathrm{a}, 6 \mathrm{~b}$ protons correlate with the carbonyl groups on the same sites which, in turn, correlate with the respective methyl protons. The proton $J$-coupling patterns were found to be similar to those in the unsubstituted systems. The acetyl on the $\mathrm{O} 2$-site in the distorted glucopyranose $\operatorname{ring}(\mathrm{s})$ is in proximity to $\mathrm{H}-5$ and hence pseudo axial, making $\mathrm{H}-2$ pseudo equatorial, on the same site. The same extent of average distortion can also be found in alkylated, benzylated and benzoylated CDs. $^{33}$ The stereochemical distortions found in solution are similar to the symmetry perturbations detected in CDs bearing benzoyl, at least on the secondary sites. These distortions were explained by the loss of the strong hydrogen-bond network on the secondary sites of the adjacent units and the need to minimize the repulsive steric interactions between the bulk substituents. Glucopyranoside units are found in an intermediate conformation, as they are between the two chairs. These ring deformations affect some units only and the undistorted ${ }^{4} \mathrm{C}_{1}$ chairs were simultaneously detected. The truncated cone shape is heavily perturbed because of these distortions and the polarity differences between the outside and the inside of the cavity can be affected significantly, as can the solubility of derivatized CDs and their complexation properties. Similar conformations have been found in both $\mathrm{CDCl}_{3}$ and $\mathrm{CD}_{3} \mathrm{OD} .^{1}$

Complex formation between a structural analogue, 1,4-bis(2-ethylhexyl)benzene-1,2-dicarboxylate (our model compound is 1,4-dicarboxylate), and perbenzoylated $\beta \mathrm{CD}$ has been investigated by Yu et al., ${ }^{34}$ who concluded that the host-guest complex was stabilized by $\pi-\pi$ interactions between the aromatic ester and the perbenzoylated $\beta \mathrm{CD}$.

In this paper, we report our studies on the interaction between a branched alkyl chain ester of terephthalic acid and peracetylated CDs. The presence of incompletely and possibly inhomogeneously dried products - due either to the drying procedure or the adsorption of small amounts of water from the air - is to be expected when bulk quantities are handled. It is worth pointing out that polar solvent molecules can be retained within the inner cavity of native CDs. Molecular dynamics evidence that the average residence time for residual DMSO lies in the nanosecond range, which is at least one order of magnitude longer than the one found for water. ${ }^{35}$ Molecular dynamics was also used to study the solvent polarity effect on the formation of CD dimers and emphasized the importance of hydrogen bonds in the formation. Although, it is also true that in the simulation the authors used solvents too, in which the native CDs are practically insoluble, and/or form complexes. Losing the strong hydrogen bonds by the substitution of the electrostatic and lipophilic interactions become dominant. ${ }^{36}$ Having noticed the difficulties of water removal from the lipophilic CDs, the possible effect of water on solution complexation in the nonpolar solvent, $\mathrm{CDCl}_{3}$, was also investigated.

\section{Experimental}

\subsection{Materials}

Acetic anhydride, methanol, $\mathrm{FeCl}_{3} \cdot 6 \mathrm{H}_{2} \mathrm{O}$ and methylene chloride were purchased from Sigma-Aldrich, while $\alpha$-, $\beta$ - and $\gamma$ CDs were generous gifts from Roquette, France. $\mathrm{CDCl}_{3}$ is a product of EurIsotop (D007H). Racemic 1,4-bis(2-ethylhexyl)benzene-1,4dicarboxylate was obtained from Giganplast, Italy.

\subsection{Methods}

2.2.1. ${ }^{1} \mathrm{H}$ NMR measurements. Water-saturated $\mathrm{CDCl}_{3}$ was prepared by shaking $\mathrm{CDCl}_{3}(10 \mathrm{ml})$ and water $(2 \mathrm{ml})$ for $5 \mathrm{~min}$ and then allowed to separate overnight. Molar ratios of water in 
the $\mathrm{CDCl}_{3}$ solutions of peracetyl CDs were calculated from the integrals of the anomeric proton signals of cyclodextrin and the water protons.

NMR stock solutions were prepared in $\mathrm{CDCl}_{3}$ at fixed 16-25 \pm $1 \mathrm{mM}$ concentrations (except for water complexation experiments) and the guest was added via a microliter syringe. In order to determine apparent complex stability constants, CD concentrations were kept almost constant to avoid concentration dependent shift drifts. The dried peracetylated CDs were weighed and added gradually to the water saturated $\mathrm{CDCl}_{3}$ solution in the aqueous experiments.

NMR spectra were recorded on a Bruker Avance $300 \mathrm{MHz}$ at $22.6 \pm 0.1{ }^{\circ} \mathrm{C}$. Residual chloroform of $\mathrm{CDCl}_{3}$ was used as an internal standard and chemical shifts were calibrated to the residual $\mathrm{CHCl}_{3}(\delta H=7.260)$. Chemical shifts $(\delta)$ are given in ppm.

To reduce the possible errors, pure components were always recorded at the beginning of the experiments in each series. All experiments of the same run had been recorded on same days without interruption, using 64 transients, in a $4496.40 \mathrm{~Hz}$ spectral width. The ${ }^{1} \mathrm{H}$ NMR data were processed using an ACD/NMR Processor, ${ }^{42}$ using a 4-fold zero filling and LorentzianGaussian window function $(\mathrm{LB}=-0.3, \mathrm{GF}=0.3$ ) prior to Fourier transformations.

\subsection{Synthesis}

2.3.1. Peracetylated cyclodextrins. Dried CDs $(10 \mathrm{mmol}$, 9.7-13 g) were acetylated in acetic anhydride using $\mathrm{FeCl}_{3} \cdot 6 \mathrm{H}_{2} \mathrm{O}$ as a catalyst (10 mol\%), according to the published method, ${ }^{37}$ and recrystallized twice from $\mathrm{MeOH}$ prior to use. The obtained materials, PAc $\alpha \mathrm{CD}$ (16 g, 94\%), PAc $\beta \mathrm{CD}(16 \mathrm{~g}, 80 \%)$ and PAc $\gamma \mathrm{CD}$ (20 g, 88\%), were attempted to dry as described in the next section.

2.3.2. Drying of peracetylated cyclodextrins. The CD derivatives were subjected to further drying to remove most of the water present by means of the following procedures: (a) room temperature, air, 3 days; (b) $80{ }^{\circ} \mathrm{C}$, air, 3 days; (c) $80{ }^{\circ} \mathrm{C}$, vacuum, in the presence of $\mathrm{P}_{2} \mathrm{O}_{5}$ and $\mathrm{KOH}, 3$ days; (d) peracetyl $\mathrm{CD}(10 \mathrm{~g})$ was dissolved in methylene chloride $(10 \mathrm{ml})$ and dried with sodium sulfate $(1 \mathrm{~g})$, filtered and rotoevaporated. The solid was dried at $80{ }^{\circ} \mathrm{C}$ for 3 days. (e) From peracetyl CD ( $2 \mathrm{~g}$ ) by azeotropic distillations: toluene $(2 \times 10 \mathrm{ml})$, then methanol $(3 \times 20 \mathrm{ml})$, and finally ethanol free chloroform $(2 \times 20 \mathrm{ml})$ were dried at $80{ }^{\circ} \mathrm{C}$ at 2 mbar.

\section{Results and discussion}

\subsection{Peracetyl cyclodextrin-water systems}

The X-ray structure of peracetylated $\alpha \mathrm{CD}$ contains two moles of water as reported by Harata. ${ }^{3}$ One of these water molecules is found inside the CD cavity at the primary rim, which is not surprising considering that the acetyl groups introduced to the macrocycle reversed its lipophilicity and that carbonyl oxygens can stabilize the water in an apolar environment via hydrogen bonding. Harata, unlike Caira with $\mathrm{PAc} \gamma \mathrm{CD},{ }^{2}$ was able to prepare pure water-PAc $\alpha \mathrm{CD}$ associates although he crystallized PAc $\alpha \mathrm{CD}$ from $80 \%$ aqueous 2-propanol.

NMR measurements show that even dried peracetylated CDs (Scheme 1a) contain water. The weight percentage content of water is $1-3 \%$ in the air-dried products, which is in the 1-2 molar equivalent range of peracetylated CDs due to the ca. 95-130-fold difference in molecular weight between peracetylated CDs and water. It was found that simple heat assisted drying did not usually result in water-free materials. It was possible to completely dry peracetylated $\alpha$ - and $\beta$ CDs by azeotropic distillations. However, a relatively large amount of organic solvents used means that this method is not feasible for drying in bulk quantities. PAc $\gamma$ CD showed $\sim 1$ mol residual water after treatment. Air-dried compounds were used to study the effects of CD concentration on water chemical shifts for the NMR titrations of 1,4-bis(2ethylhexyl)benzene-1,4-dicarboxylate (Scheme 1b). It was also found that the water content did not considerably affect NMR analyses, except for PAc $\gamma \mathrm{CD}$ which produced overlapping water and guest methine proton signals. The water signals smoothly drifted with increasing guest concentration and finally moved away from the methine signal.

From the experimental data of 2-ethylhexyl benzoate, ${ }^{38}$ the methyl and some methylene protons from the guest molecule could be tentatively assigned, which enabled us to use their chemical shifts for our determination of complex composition and calculation of apparent complex stability constants. H-6 protons practically gave single signals in PAc $\alpha C D$, while the methylene protons were split and well separated in both PAc $\beta C D$
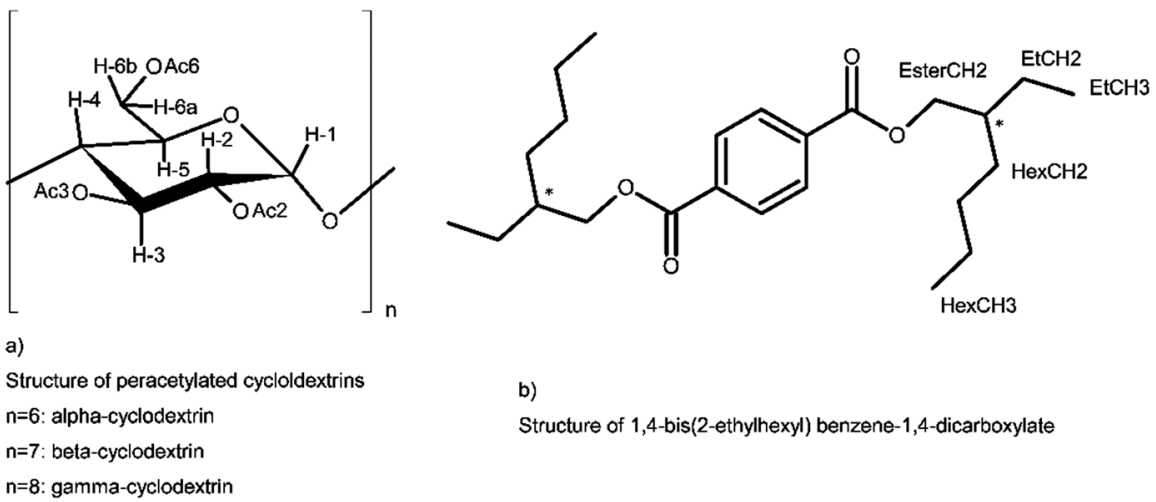

b)

Structure of 1,4-bis(2-ethylhexyl) benzene-1,4-dicarboxylate

Scheme 1 Structures of host and guest and labels for NMR identification. 


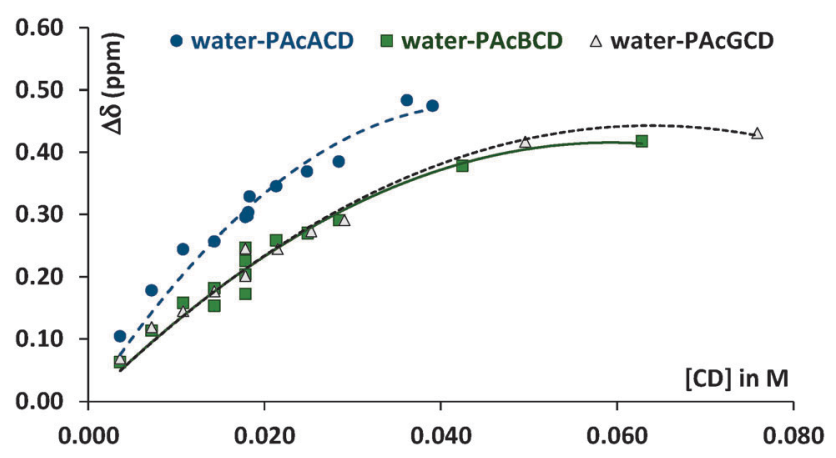

Fig. 1 Movement of water proton signals upon increasing CD concentration.

and PAc $\gamma \mathrm{CD}$ (H-6a and H-6b). Overlapping signals were also observed in the $\mathrm{H}-5$ protons of peracetyl $\alpha \mathrm{CD}$, the $\mathrm{H}-6 \mathrm{a}$ protons of peracetyl $\beta$ - and $\gamma$-CDs and the ester methylene protons of the guest. Despite these overlapping signals, the chemical shifts of $\mathrm{H}-5, \mathrm{H}-6 \mathrm{a}$ and the ester methylene protons were determined, but with relatively large errors. In worst cases, the methyl and methylene protons of the 2-ethylhexyl moiety, the most separated signals were used instead of the multiplet chemical shifts. Although, this method is not absolutely correct because it assumes that the coupling constants remain constant upon the noncovalent interaction of the molecules. Changes in coupling constants may also be indicative of complexation, ${ }^{39}$ but now they are in the range of experimental errors at the NMR frequency used.

3.1.1. Water protons. $\mathrm{CDCl}_{3}$ was saturated with water and the concentrations of the peracetylated CDs were gradually increased. Constant downfield shifts were observed in the water protons with increasing CD molar concentration, as seen in Fig. 1. The curves follow saturation-like curves. However, it is necessary to note that the last point overlaps the acetyl protons and so an exact shift cannot be determined. It was therefore set as being equal to the acetyl protons and the uncertain points fit into the trends well.

Water proton chemical shift differences $(\Delta \delta)$ show larger deviances at almost identical peracetylated CD molar concentrations, but at different molar fractions. This is probably due to experimental weaknesses, such as uncertainties in weighing and changing sample concentrations upon the transfer of solutions to the tubes. There are enough points to obtain a trend for PAc $\alpha \mathrm{CD}$, but the measurements still show large deviations, as seen in Fig. 2.

Water signals moved and finally overlapped with the acetyl signals of the CD derivative and this fact prevented the exact determination of the water content at high $\mathrm{CD} /$ water ratios. This also means that the absence or presence of water cannot be seen at high peracetyl $\mathrm{CD}$-water molar ratios. When water was replaced with deuterium oxide, the HDO signals moved away from the acetyl proton signals, in PAc $\alpha \mathrm{CD}$ and PAc $\beta C D$, but not in PAc $\gamma \mathrm{CD}$ (see the ESI $\dagger$ ). The water content of $\mathrm{CDCl}_{3}$ $(\leq 0.01 \%)$ is in the same molar range as the CDs. Owing to ambiguous water content in the peracetylated CDs, the water content of the test solutions and the water content of "absolute" $\mathrm{CDCl}_{3}$, the boundary conditions of Job's method (constant sum of molar concentrations) could not be fulfilled.

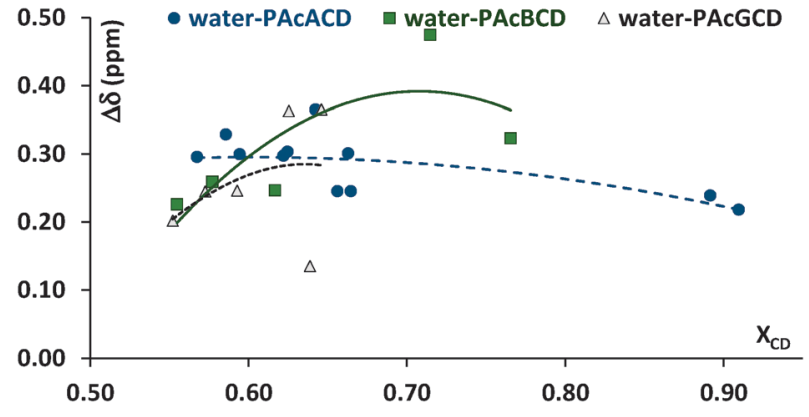

Fig. 2 Water proton chemical shift differences at almost identical molar concentrations but different peracetylated CD molar fractions.

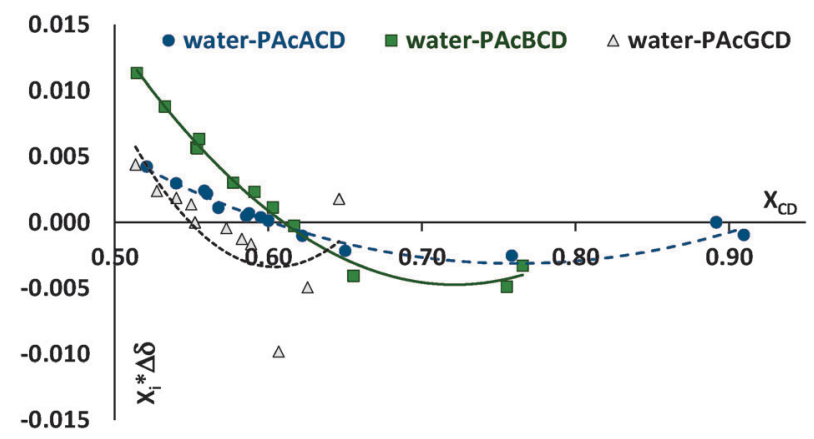

Fig. 3 Job's plot-like graph of water proton shift differences as a function of water molar fraction.

However, Job's plot-like chart can be created despite these weaknesses $^{8-10,40}$ (Fig. 3). When Job's plot is created at a nonconstant sum of moles, the extremity is shifted to higher host molar fractions.

Considering this effect we concluded the existence of an almost equimolar association between water and peracetylated CDs. This is also confirmed by the nonlinear curve fitting and stability constant calculations.

3.1.2. Cyclodextrin protons. A tentative assignment of the acetyl methyl protons of peracetyl $-\alpha$ - and $-\gamma$ CDs is based on the assignment of peracetyl- $\beta C D$ published by Uccello-Barretta et al. ${ }^{1}$ Although it appears that the acetyl protons are involved in the complexation of water and the phthalate ester (see later), unambiguous assignments are not vital at the secondary rim of CDs. CD chemical shifts show small changes with CD molar concentration without any recognizable trends. These changes in all protons are in the range of 0.003-0.005 ppm over $0.03 \mathrm{M}^{-1}$, and almost twice that of below $0.002 \mathrm{M}^{-1}$. Concentration dependent curves seem to be linear when plotted against chemical shift changes as a function of $\mathrm{CD}$ concentration but for molar fractions the quadratic curves usually fit better. Some demonstrative examples are seen in Fig. 4. These results indicate the existence of dynamic changes in CD structures in chloroform that are triggered by complexed water. This is in accordance with the Uccello-Barretta et al. publication on peracetylated $\beta \mathrm{CD}{ }^{1}$

In $\mathrm{PAc} \alpha \mathrm{CD}$, methylene (H-6) protons are indistinguishable at low $\mathrm{CD} /$ high water concentrations. As the $\mathrm{CD} /$ water ratio decreased, 


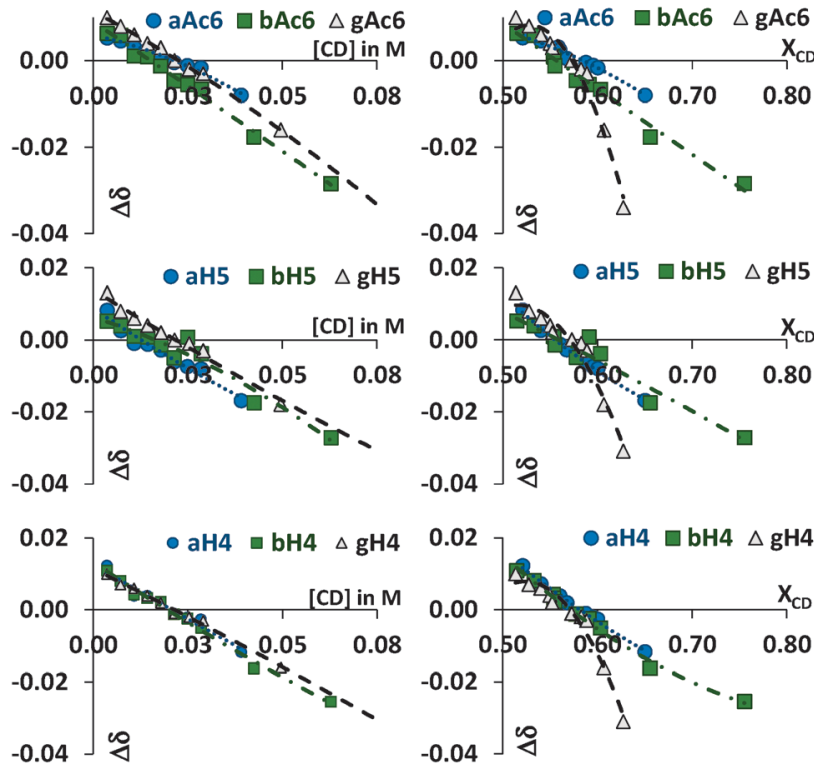

Fig. 4 Plots of selected chemical shift differences vs. molar concentration and vs. molar fraction of peracetylated CDs $(a=$ alpha, $b=$ beta, $\mathrm{g}=$ gamma $C D$, numbers refer to the appropriate proton as shown in Scheme 1a).

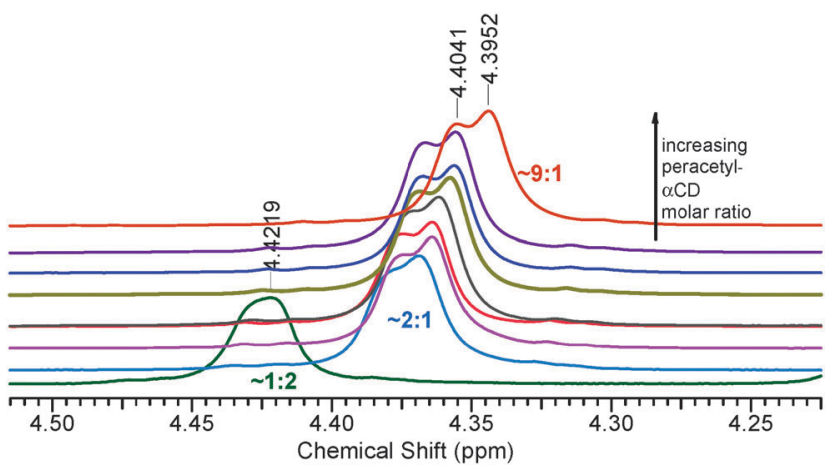

Fig. 5 Split of methylene protons in PAc $\alpha C D$ when increasing the $C D$ : water molar ratio.

at first shoulder appeared only then the signal was divided into two overlapping peaks, as seen in Fig. 5 .

$\mathrm{CD}$ : water molar ratio values were found to be at around 0.4 in our samples. The high chemical shift drift in the $\mathrm{mM}$ concentration range indicated the presence of a strong interaction, according to the theoretical complex calculations by Fielding. ${ }^{5}$ The apparent $1: 1$ complex stability constants are in the range of $10^{1}-10^{2} \mathrm{M}^{-1}$.

The a priori determination of complex stoichiometry is not necessary if we use the nonlinear curve fitting procedure. WinEQNMR2 $2^{6,41}$ is a suitable program for calculating $1: 1,1: 2$ and $2: 1$ complex stability constants from NMR data. Though this program cannot calculate higher host:guest stoichiometries, however the peracetyl- $\alpha$ - and $-\gamma \mathrm{CD}$ X-ray data suggest that these compositions are sufficient for the calculations. The goodness of parameters obtained from a nonlinear curve fitting is always challenging, particularly when the experiment number is limited.
Table 1 Calculated 1:1 complex stability constants for peracetyl $\mathrm{CD}$-water systems in $\mathrm{CDCl}_{3}$

\begin{tabular}{|c|c|c|c|}
\hline & $\mathrm{K} 11\left[\mathrm{M}^{-1}\right]$ & $\begin{array}{l}\text { Estimated } \\
\text { uncomplexed } \\
\text { water shift }^{a}\end{array}$ & $\begin{array}{l}\text { Estimated } \\
\text { complexed } \\
\text { water shift }\end{array}$ \\
\hline Peracetyl- $\alpha C D$ & $114.3 \pm 31.2$ & $1.494 \pm 0.029$ & $2.125 \pm 0.036$ \\
\hline Peracetyl- $\beta C D$ & $94.2 \pm 14.1$ & $1.523 \pm 0.015$ & $2.061 \pm 0.014$ \\
\hline Peracetyl- $\gamma$ CD & $58.2 \pm 9.6$ & $1.545 \pm 0.032$ & $2.234 \pm 0.068$ \\
\hline
\end{tabular}

Only the 1:1 peracetyl CD-water complexes in $\mathrm{CDCl}_{3}$ gave results that fulfill acceptance criteria. The calculated stability constants are tabulated in Table 1. Calculated concentrations and chemical shift are given in the ESI, $\uparrow$ Fig. S3.1 and S3.2. Values for $1: 2$ and $2: 1$ complexes were in the range of thousands. However, both their relative errors and poor estimations of uncomplexed water shifts suggest a much lower probability that such associations form.

\subsection{1,4-Bis(2-ethylhexyl)benzene-1,4-dicarboxylate complex}

The two-arm structure of the ester allows two CDs to interact with the guest molecule. Possible association modes are depicted in Scheme 2, where the additional rings represent the macrocycle being enlarged by the acetyl substituents. The free rotation of the ester moiety means that the longer alkyl chain can also approach closer to the macrocycle cavity, while the coordination of the second CD is sterically more hindered in this case. The molecular dimensions of the peracetyl $\gamma \mathrm{CD}$ allow for deeper intrusion into the cyclodextrin cavity and even the complete cross is geometrically allowed.

3.2.1. Water protons. The water signal showed a downfield movement with increasing guest concentration in the case of PAc $\alpha C D$ and PAc $\beta C D$, while the opposite movement was detected in the PAc $\gamma \mathrm{CD}$ analogue. This would appear to suggest that water is partially, but continuously, excluded from PAc $\gamma \mathrm{CD}$, while two other CDs may participate in a three component complexation process with $\mathrm{CD}$, water molecules and the guest. In all cases, the maximal chemical shift changes were at around $0.1 \mathrm{ppm}$, while water signals finally overlapped with the $\mathrm{CH}$ signals of the ester in PAc $\gamma \mathrm{CD}$. The dried PAc $\gamma \mathrm{CD}$ still contained equimolar water, which together with the water content of the $\mathrm{CDCl}_{3}$ was a competitor for the guest molecule as seen in Fig. 6.

A section of proton spectra is shown in Fig. 7 in order to demonstrate water proton movements as a function of the host: guest ratio.

Calculation of complex stability constants for water in our three-component systems (neglecting the solvent) gave very high errors in all cases, as can be concluded from Table 2 . The peracetylated cyclodextrins contain water and in the complexation experiments, the water concentrations varied in a very tight range. Additionally, in the case of PAc $\gamma \mathrm{CD}$ due to the water signal overlaps with the methine signal of the organic guest and its chemical shifts are uncertain and integration is not satisfactory. For the PAc $\alpha \mathrm{CD}$ case overlapping water and Ac3 


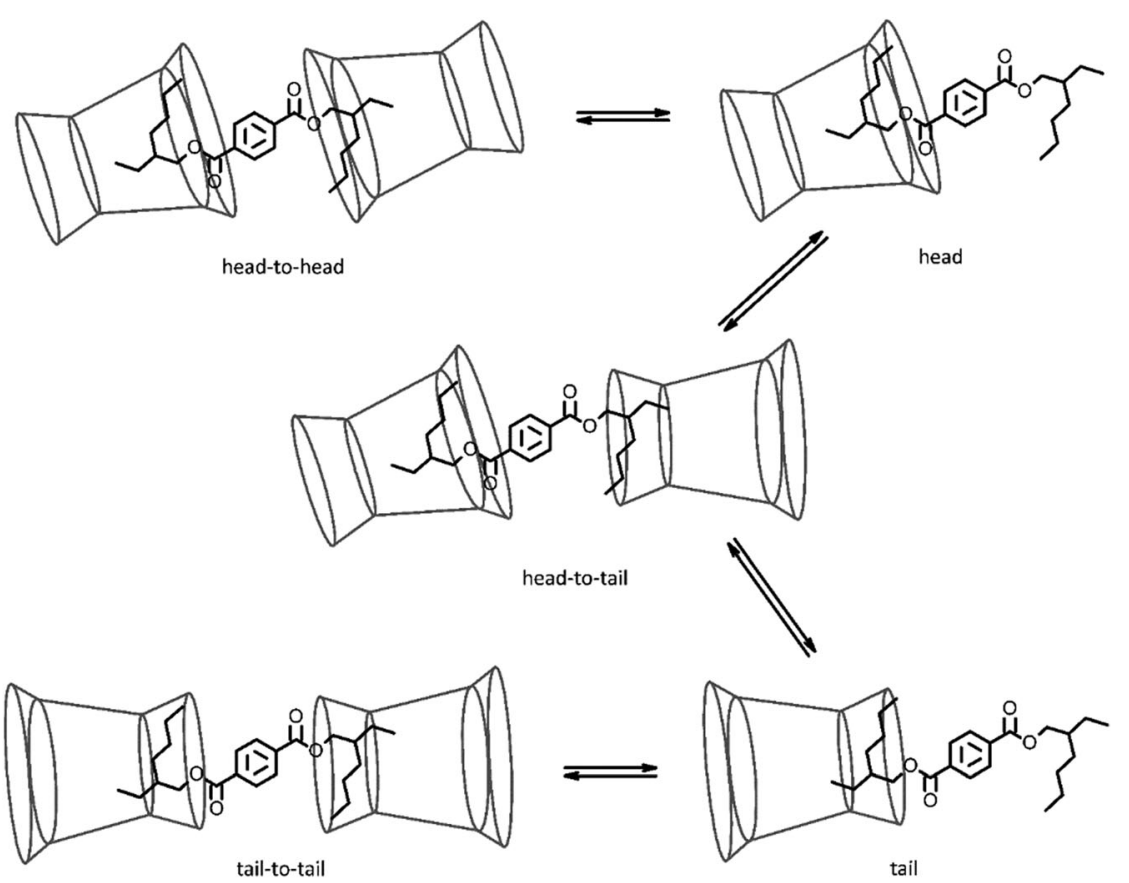

Scheme 2 Possible structures for various peracetylated CDs and 1,4-bis(2-ethylhexyl)benzene-1,4-dicarboxylate complexes.

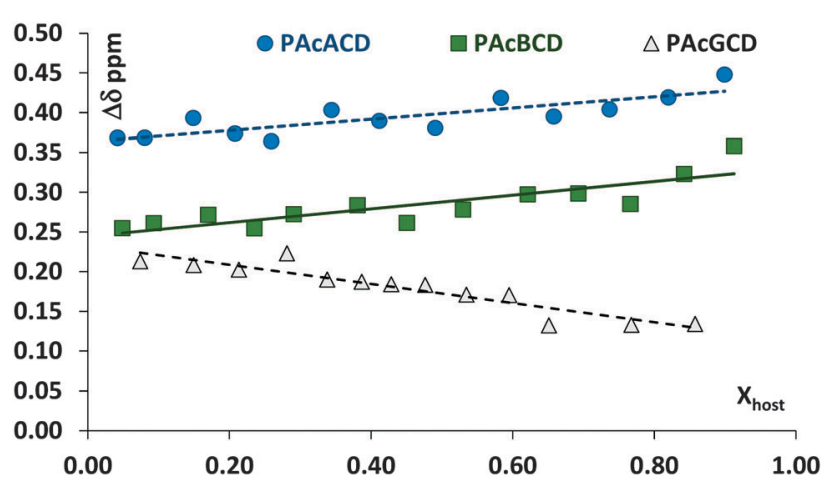

Fig. 6 Water proton signal movement as a function of the molar ratio.

methyl signals cause similar difficulties. Because the estimated stability constants are out of acceptance, the complexed/free water ratio cannot be well estimated. From Fig. 6 it can be concluded that the guest molecule cannot exclude water from PAc $\alpha C D$ and PA $\beta C D$, the lower limit of $1: 1$ apparent stability constant is at around $100 \mathrm{M}^{-1}$, while for the PA $\gamma \mathrm{CD}$ system the upper limit is around $200 \mathrm{M}^{-1}$. These values are in good agreement with the values of Table 3 for PAc $\alpha$-and - $\beta$ CDs.

3.2.2. Cyclodextrin protons. While the protons outside the cavity ( $\mathrm{H}-1, \mathrm{H}-2, \mathrm{H}-4$, and often $\mathrm{H}-6)$ usually show negligible shift changes in natural or partially substituted CDs due to their more rigid structures, in peracetylated CDs these protons also reflect structural changes upon complexation. At the same time, the widened cavity can reduce the environmental changes in protons close to the cavity. Hydrophobic substituents, like methyl or acetyl or generally, alkyl- and alkylcarbonyl groups, virtually inverse the polarity of the CD cavity due to their apolar character and the cavities favor the more polar groups in an

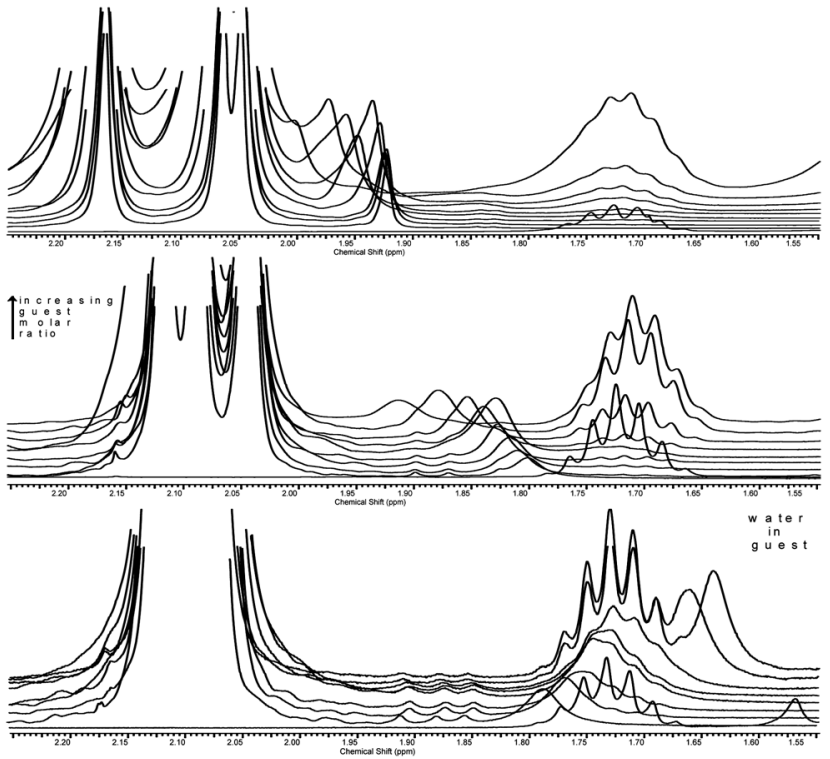

Fig. 7 Water proton signal movement upon increasing the guest molecule molar ratio. Top: PerAc $\alpha C D$, middle: PerAc $\beta C D$, bottom: PerAc $\gamma C D$. Guest molar ratio increases from bottom to top in each spectrum; some curves have been removed to improve visibility.

apolar environment. The wider cavities allow for interaction with larger molecules, e.g. those that contain branched alkyl chains or structural isomers of aromatic compounds.

Almost all CD protons moved, but usually without characteristic extremities in Job's plots, upon changing the concentration of the host molecule. It is assumed that these missing extremities are due to the opposite movement or the large distances between interacting and non-interacting moieties. 
Table 2 Calculated 1:1 complex stability constants for peracetyl CDwater-1,4-bis(2-ethylhexyl)benzene-1,4-dicarboxylate systems in $\mathrm{CDCl}_{3}$

\begin{tabular}{lcl}
\hline & & Estimated \\
& $\mathrm{K} 11\left[\mathrm{M}^{-1}\right]$ & $\mathrm{K} 11\left[\mathrm{M}^{-1}\right]$ (based on Table 3) \\
\hline Peracetyl- $\alpha \mathrm{CD}$ & $4.1 \pm 38.2$ & $>93$ (lower limit) \\
Peracetyl- $\beta \mathrm{CD}$ & $6.2 \pm 4.6$ & $>111$ (lower limit) \\
Peracetyl- $\gamma \mathrm{CD}$ & $34.2 \pm 37.6$ & $<182$ (upper limit)
\end{tabular}

The reproducibility of chemical shifts at identical concentrations was typically in the 0.001-0.004 range. Although PAc $\alpha C D$ $\mathrm{H}-5$ protons overlap the ester methylene protons, it was possible to determine individual chemical shifts in most cases. This was not true for PAc $\beta C D$ and PAc $\gamma C D$ where the H-6a protons completely overlapped the ester methylene protons at a higher CD molar ratio and it was impossible to determine the individual chemical shifts.

Although the acetyl protons are in huge excess in the CDs and their methyl groups can be very far from each other, the outcome of their shift changes are above experimental errors, as can be seen in their Job's plots, Fig. 8. The curves show large fluctuations, at ester molar fractions of around 0.25-0.3, 0.45-0.5 and 0.67 , while definite minima - corresponding to $2: 1$ (or $3: 1$, due to inherent uncertainty in Job's plot), $1: 1$ and $1: 2$ host:guest ratios - can be found in the case of PAc $\alpha \mathrm{CD}$ and PAc $\beta C D$. Only one minimum is found for PAc $\gamma \mathrm{CD}$ at $\sim 0.3$ guest molar fraction. The acetyl groups are far from the cavity and the observed chemical shift drifts can give information about the host and guest interaction centers. In the case of PAc $\alpha \mathrm{CD}$, the secondary acetyl substituents show an almost equal but smaller interaction than the primary rim substituent, where the $0.25-0.3$ molar ratio minimum is missing. An interaction between the host and guest is therefore assumed to exist at the secondary rim, while the extremities point to a secondary rim interaction for $1: 1$, and a head-to-head association for the 2:1 host: guest ratio. PAc $\beta C D$ shows almost equal participation from the $\mathrm{O}(3)$ - and $\mathrm{O}(6)$ acetyl groups in complexation, while the largest differences can be found in all PAc $\gamma \mathrm{CD}$ acetyl groups which affect both sides of the host molecule. The dimensions of both molecules allow the guest to pass through the CD cavity in the latter case. This can also be confirmed by the migration of water protons in the opposite direction suggesting that the ester is able to move water from a relatively polar environment to a less polar one. NOESY and/or ROESY experiments may have given a more realistic picture, however these experiments failed due to the high number of almost chemically equivalent protons combined with the chirality of the ester.

3.2.3. Guest protons. The phthalate ester has two arms that are potential targets for complexation. It is a priori assumed

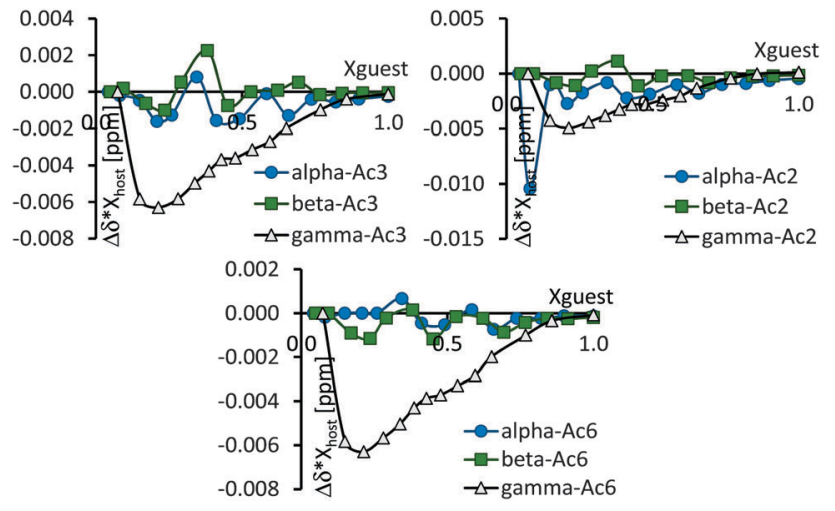

Fig. 8 Job's plot of CD acetyl methyl protons.

that both alkyl chains can be complexed at low guest molar ratios giving 2:1 host: guest associations. The 1:1 complex became dominant upon increasing guest concentration, as is illustrated in Scheme 2. The branched alkyl chain can penetrate deeper into the $\mathrm{CD}$ cavity allowing the more polar carbonyl group(s) to interact with the similar CD groups. Unfortunately, the guest molecule's most sensitive proton signals, the ester methylene and branch methine proton, overlap with some CD signals and also with water signals, in the case of PAc $\gamma \mathrm{CD}$. Additionally, the host methine group's chiral center brings new complexities as a potential enantiomer-selective complexation. Chemical shift determinations are ambiguous as a consequence of these bad constellations and, as such, conclusions should be handled carefully. Furthermore, CD Job's plots do not completely confirm the a priori assumed association stoichiometry. Job's plots of ester methylene and the methine protons can be seen in Fig. 9.

The aromatic region of the guest molecule is the only part of the molecule which has no signals that overlap with $\mathrm{CD}$ and water protons. Job's plot created from the data given in Fig. 10 is not absolutely clear, however the signal shape of the aromatic protons shows essential changes upon host: guest molar ratio changes, as is demonstrated in Fig. 11. The large extremities at the $\sim 0.3$ molar ratio of the guest suggest $2: 1 \mathrm{CD}$ : ester associations for PAc $\alpha \mathrm{CD}$ and PAc $\beta C D$. In the PAc $\gamma \mathrm{CD}$ case, where water can be more readily excluded, a minimum can be found at around the 1:1 molar ratio. Water exclusion by the host is also confirmed by the higher complex stability constants seen in Table 3. The reasonable 1:2 host guest ratio also supports the idea that the terephthalate ester can pass through the PAc $\gamma \mathrm{CD}$ cavity. The weak minima, which rather seem to be shoulders in the other two cases, suggest the existence of the

Table 3 Calculated stability constants from the aromatic proton shifts of different complex models (numbers after $\mathrm{K}$ shows the host : guest molar ratio)

\begin{tabular}{|c|c|c|c|c|c|}
\hline & $\mathrm{K} 11^{a}\left[\mathrm{M}^{-1}\right]$ & $\mathrm{K} 11^{b}\left[\mathrm{M}^{-1}\right]$ & $\mathrm{K} 12^{b}\left[{ }^{\mathrm{M}-2}\right]$ & $\mathrm{K} 11^{c}\left[\mathrm{M}^{-1}\right]$ & $\mathrm{K} 21^{c}\left[\mathrm{M}^{-2}\right]$ \\
\hline Peracetyl- $\beta$ CD & $114.8 \pm 3.0$ & $28.3 \pm 1.6$ & $51.3 \pm 2.0$ & $37.7 \pm 1.5$ & $25.4 \pm 10.5$ \\
\hline
\end{tabular}

${ }^{a}$ The results are from the $1: 1$ (host: guest) curve fitting procedure. ${ }^{b}$ The results are from the $1: 2$ (host: guest) curve fitting procedure. ${ }^{c}$ The results are from the $2: 1$ (host: guest) curve fitting procedure. 

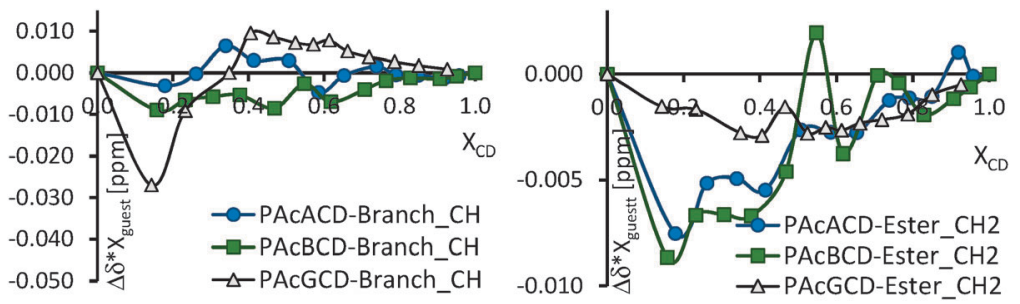

Fig. 9 Job's plot of guest methine and ester methylene protons.

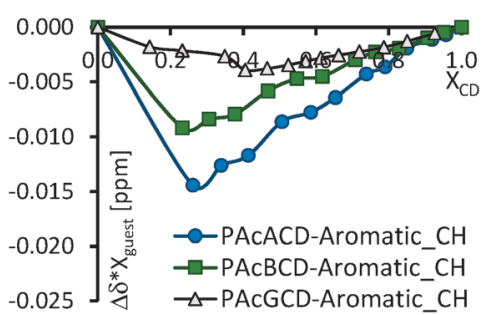

Fig. 10 Job's plot of aromatic protons.

1:1 and 1:2 host: guest associations for the PAc $\gamma \mathrm{CD}$ and $1: 1$ and $2: 1$ for the other two CDs. Only loose associations can be made from all figures. According to the literature, ${ }^{5}$ artificial complex simulations gave the apparent complex stability constants to be in the $10-100 \mathrm{M}^{-1}$ range. To calculate the stability constants, the three-component systems were reduced to two components as water was neglected and the multicomponent curve fitting procedure from WinEQNMR2 $2^{6,41}$ was used.

The stability constants calculated from the aromatic protons of the guest are summarized in Table 3. Simulated concentration curves and chemical shifts can be found in the ESI, $\dagger$ Fig. S3.3 and S3.4. Calculations for other than ester aromatic protons failed to give reasonable results, which is partially the consequence of the relatively large error in the chemical shifts of the overlapped signals in the complex concentration range. An additional source of the failed calculations can be found from the fact that the two identical arms of the guest the 1:1,1:2 and 2:1 host: guest ratios are practically indistinguishable in the alkyl region.
The calculated stability constants show that $1: 1$ and $2: 1$ complexes are the most probable associations in the case of PAc $\alpha C D$, while $1: 1$ and both $1: 2$ and $2: 1$ associations for PAc $\beta C D$ and $\mathrm{PAc} \gamma \mathrm{CD}$ are reasonable but in these cases, the $1: 1$ association is the most probable. These results are also in accordance with the molecular dimensions of CDs. The large error suggests that the $1: 2$ complex, for PAc $\alpha \mathrm{CD}$, and $1: 2$ and $2: 1$ complexes, for $\mathrm{PAc} \gamma \mathrm{CD}$, are less adequate models for the association by handling the peracetyl CD-water as one unit.

Similar calculations for the CD protons pointed to the glucose units participating differently in the complexation and showed considerable geometrical changes upon association. Data for the calculations can be found in the ESI. $\dagger$

\section{Conclusions}

Water interacts strongly with the polar moieties of peracetyl CDs. The water content of peracetylated CDs can control the complexation of apolar organic molecules. Although the limited solubility of water in chloroform results in very low water content in the solvent, this residual amount of water is still in the equimolar range, as compared to the low dissolved CD concentration. Complete water removal from the peracetylated CDs is difficult and far from feasibility in bulk quantities. Approximately equimolar amounts of water remained in peracetyl- $\gamma$ CD even after azeotropic removal, which was the only successful method for $\alpha$ - and $\beta$ CDs. The water signal at higher CD : water molar ratios

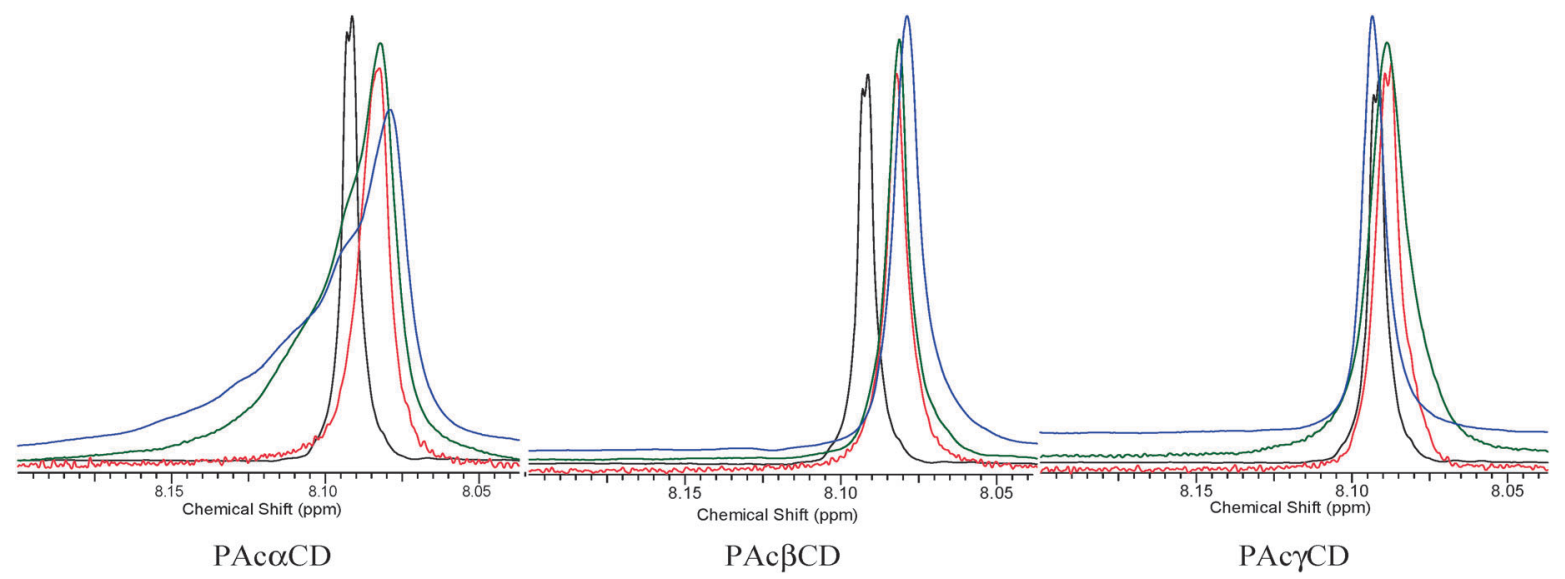

Fig. 11 Aromatic protons at low, medium, and high guest ratios (black: no CD, red: low, green: middle, blue: high peracetyl CD : guest molar ratio, respectively). 
overlapped with the acetyl methyl signals. Complexation means that the water content of chloroform can be considerably higher than when without peracetylated CDs.

Host and guest proton signals, and often water signals, overlap which made the determination of the chemical shifts of the interacting moieties difficult and prone to large relative errors. Structural changes in CDs upon interaction with the guest molecule affect all CD protons, regardless of their spatial interactions in the complexation process. The water proton NMR signal showed different shift directions upon increasing the aromatic ester molar ratio: a downfield shift in the cases of PAc $\alpha C D$ and PAc $\beta C D$ and an upfield shift in the case of PAc $\gamma \mathrm{CD}$.

The interaction between apolar CD derivatives and polar (water) or apolar guests (1,4-bis(2-ethylhexyl)benzene-1,4-dicarboxylate) was confirmed via NMR. The peracetyl CD-water complexes are dominantly found in a 1:1 molar ratio with moderate complex stability constants. Both peracetyl- $\alpha$ - and $-\beta C D$ s seem to form three component complexes with $1: 1,1: 2$, and $2: 1$ molecular ratios between CDs and esters, while the guest can exclude water from the peracetyl- $\gamma \mathrm{CD}$ cavity by forming dominantly $1: 1$ molecular associations.

\section{Acknowledgements}

This research was kindly funded by the European Community's Seventh Framework Program through the EU project MAPSYN: Microwave, Acoustic and Plasma Syntheses, under grant agreement No. CP-IP 309376. P. Cintas thanks the Ministry of Economy and Competitiveness (Project CTQ2013-44787-P) and the Junta de Extremadura-FEDER (Grant GR15022) for financial support. Financial support from Resilia SRL (Samarate, Italy) is acknowledged. The authors acknowledge the assistance and suggestions of Orsolya Töke (MTA-TKK, Budapest, Hungary) in NMR experiments, and the invaluable help of Éva Fenyvesi (Cyclolab Ltd, Budapest, Hungary) in manuscript preparation.

\section{References}

1 G. Uccello-Barretta, G. Sicoli, F. Balzano and P. Salvadori, Carbohydr. Res., 2003, 338, 1103-1107.

2 M. R. Caira, G. Bettinetti, M. Sorrenti, L. Catenacci, D. Cruickshank and K. Davies, Chem. Commun., 2007, 1221-1223.

3 K. Harata, Chem. Lett., 1998, 589-590.

4 M. Añibarro, K. Gessler, I. Uson, G. M. Sheldrick, K. Harata, K. Uekama, F. Hirayama, Y. Abe and W. Saenger, J. Am. Chem. Soc., 2001, 123, 11854-11862.

5 L. Fielding, Tetrahedron, 2000, 56, 6151-6170.

6 M. J. Hynes, J. Chem. Soc., Dalton Trans., 1993, 311-312.

7 G. Uccello-Barretta, L. Vanni and F. Balzano, J. Chromatogr. A, 2010, 1217, 928-940.

8 H. Dodziuk, W. Kozminski and A. Ejchart, Chirality, 2004, 16, 90-105.
9 B. Chankvetadze, G. Endresz and G. Blaschke, Chem. Soc. Rev., 1996, 25, 141-153.

10 H. J. Schneider, F. Hacket and V. Rüdiger, Chem. Rev., 1998, 98, 1755-1785.

11 B. Chankvetadze, Chem. Soc. Rev., 2004, 33, 337-347.

12 M. Karplus, J. Am. Chem. Soc., 1963, 85, 2870-2871.

13 C. Altona and M. Sundaralingam, J. Am. Chem. Soc., 1973, 95, 2333-2344.

14 J. E. H. Köhler, M. Hohla, M. Richters and W. König, Angew. Chem., Int. Ed. Engl., 1992, 31, 319-320.

15 T. Köpke, H. G. Schmarr and A. Mosandl, Flavour Fragrance J., 1992, 7, 205-211.

16 I. Abe, N. Fujimoto and T. Nakahara, J. Chromatogr. A, 1994, 676, 469-473.

17 C. Bicchi, V. Manzin, A. D’Amato and P. Rubiolo, Flavour Fragrance J., 1995, 10, 127-137.

18 K. Freudenberg and R. Jacobi, Justus Liebigs Ann. Chem., 1935, 518, 102-108.

19 M. Komiyama, H. Yamamoto and H. Hirai, Chem. Lett., 1984, 1081-1084.

20 C. Baudin, B. Perly and A. Gadelle, EP Pat., 787744 A1 970806, 1997.

21 C. Baudin, F. Tardy, J.-P. Dalbiez, C. Jankowski, C. Fajolles, G. Leclair, B. Amekraz, B. Perly and L. Mauclaire, Carbohydr. Res., 2005, 340, 131-138.

22 K. A. Kopytin, S. Y. Kudryashov and L. A. Onuchak, Russ. J. Phys. Chem. A, 2012, 86, 147-150.

23 K. Matsubara, T. Kuriki, H. Arima, K. Wakamatsu, T. Irie and K. Uekama, Minutes of the 5th International Symposium on Cyclodextrins, Paris, 1990, p. 491.

24 M. Thunhorst and U. Holzgrabe, Magn. Reson. Chem., 1998, 36, 211-216.

25 J. Zmitek, D. Fercej-Temeljotov, K. Verhnjak, S. Kotnik and M. Kovacic, EP Pat., 578231 A1, 1994.

26 T. Kitagawa, A. Okamoto, T. Kanai, K. Shibayama, T. Aoki and S. Yamakawa, EP Pat., 970936, 2000.

27 L. Kim, A.-D. Stancu, E. Diacu, H.-J. Buschmann and L. Mutihac, Supramol. Chem., 2009, 21, 131-134.

28 E. Diacu, L. Mutihac, E. Ruse and M. M. Ceausescu, J. Inclusion Phenom. Macrocyclic Chem., 2011, 71, 339-342.

29 Z. P. Onuchak, L. A. Burmatnova, T. S. Spiryaeva, E. A. Kuraeva and Y. G. Belousova, Russ. J. Phys. Chem. A, 2012, 86, 1308-1317.

30 C. M. Buchanan, D. W. Dixon, R. J. Offermann, J. Szejtli, L. Szente and M. Vikmon, Minutes of the 10th International Symposium on Cyclodextrins, Michigan, 2000, p. 586.

31 M. Y. Lee, H. S. Ganapathy and K. T. Lim, J. Phys. Chem. Solids, 2010, 71, 630-633.

32 G. Gattuso, C. Gargiulli and M. F. Parisi, Int. J. Mol. Sci., 2007, 8, 1052-1063.

33 G. Uccello-Barretta, F. Balzano, A. Cuzzola, R. Menicagli and P. Salvadori, Eur. J. Org. Chem., 2000, 449-453.

34 B. Z. Yu, J. W. Chung and S.-Y. Kwak, Environ. Sci. Technol., 2008, 42, 7522-7527.

35 J. Rodriguez, D. Hernán Rico, L. Domenianni and D. Laria, J. Phys. Chem. B, 2008, 112, 7522-7529. 
36 H. Zhang, T. Tan, W. Feng and D. van der Spoel, J. Phys. Chem. B, 2012, 116, 12684-12693.

37 L. Jicsinszky and J. Szejtli, Minutes of the 6th International Symposium on Cyclodextrins, Chicago, 1992, p. 96.

38 2-Ethylhexyl benzoate: SDBS No. 17041HSP-43-349, http:// sdbs.db.aist.go.jp. (Accessed on September, 2014).

39 S. Bekiroglu, L. Kenne and C. Sandström, J. Org. Chem., 2003, 68, 1671-1678.
40 S. Lee, D.-H. Yi and S. Jung, Bull. Korean Chem. Soc., 2004, 25, 216-220.

41 Current version of WinEQNMR (v2.0) can be obtained free of charge from Michel J. Hynes, National University of Ireland, Department of Chemistry, Galway, Ireland; michael.j.hynes@ nuigalway.ie.

42 ACD/NMR Processor Academic Edition Release 12.00 product version 12.01 (Build 39104), Advanced Chemistry Development Inc. (www.acdlabs.com). 